\title{
Factores de riesgo para cáncer de mama. Revisión de la literatura: Rol potencial de Enfermería
}

\author{
Risk factors for breast cancer. Literature Review: \\ Potential roll of Nursing
}

Mtra. Sofía Elena Pérez Zumano* Mtra. Leticia Sandoval Alonso**, Mtro Hugo Tapia Martínez ${ }^{* *}$.

*Profesora de Carrera Asociada B de Tiempo Completo ENEO UNAM. Responsable del proyecto, ** Profesora de Carrera Asociada B de Tiempo Completo. Fundamentos de Enfermería, ${ }^{\star \star \star}$ Profesor de Carrera Asociada B de Tiempo Completo. Corresponsable del proyecto.

\section{Resumen}

El cáncer de mama es la primera causa de mortalidad por neoplasias en mujeres mexicanas mayores de 25 años, es un problema de salud para la mujer, las familias y la sociedad que requiere diversas estrategias para reducir la incidencia y prevalencia. Se han identificado una serie de factores de riesgo relacionados con la historia reproductiva, estilo de vida de las mujeres y otros de origen diverso que incrementan la posibilidad de presentar la enfermedad.

En la multidimensionalidad de esta problemática Enfermería debe privilegiar la adopción de estilos de vida saludables que incidan en los factores de riesgo potencialmente modificables. Así mismo, fomentar métodos para la detección temprana: autoexploración, examen clínico y mastografía, para que la enfermedad no se presente, o sea diagnosticada en estadios más tempranos.

Palabras clave: cáncer de mama, factores de riesgo, intervención de enfermería.
The breast cancer is the first cause of mortality by cancer in Mexican women majors of 25 years, is a problem of health for the woman, the families and the society that requires diverse strategies to reduce to the incidence and prevalence. A series of risk factors related to reproductive history, life style of the women and others of diverse origin have been identified, that increases the possibility of presenting the disease. In the multi-dimensionality of this problematic Nursing it must privilege the adoption of healthful life styles that affect the potentially modifiable risk factors. Also, to foment methods for the early detection: self-exploration, clinical examination and mammography, so that the disease does not appear, that is diagnosed in earlier stages.

Key words: breast cancer, factors of risk, nursing intervention. 


\section{INTRODUCCIÓN}

El cáncer de mama (CaMa) es un problema de salud pública, actualmente es más probable que las mujeres mexicanas mueran por CaMa que por cáncer cervico uterino. Este fenómeno es multidimensional y entre otros factores es debido a la transición demográfica y epidemiológica, cambios en el estilo de vida, falta de acceso a los servicios de salud, desconocimiento de las mujeres sobre la salud de los senos, personal de salud con escasa preparación en la detección oportuna y en la interpretación de las mastografías, que influyen en la morbimortalidad.

Los avances en investigación epidemiológica y clínica de los últimos años, han permitido identificar una serie de factores de riesgo a CaMa relacionados con la historia reproductiva, estilo de vida de las mujeres y otros de origen diverso que incrementan la posibilidad de presentar la enfermedad.

Para contribuir a la atención de este problema las enfermeras pueden trabajar intervenciones focalizadas en grupos vulnerables, particularmente con los factores de riesgo potencialmente modificables para reducir la incidencia y prevalencia del CaMa. Además de alertar a las mujeres sobre los métodos de detección: autoexploración, examen clínico anual por personal de salud capacitado y la mastografía.

En este ensayo se realizó una revisión de la literatura de diversas fuentes para actualizar los conocimientos sobre el tema, que permitan a los profesionales identificar potenciales áreas de desarrollo que contribuyan a la salud de la mujer. Se analizan los aspectos más relevantes de la epidemiología del CaMa, los factores de riesgo y el rol profesional de la enfermera para modificar este problemática.

\section{Epidemiología del cáncer de mama}

El CaMa es la causa más común de muerte por cáncer en las mujeres de todo el mundo con más de 410.000 defunciones por año que representan cerca del $14 \%$ de todas las muertes debidas al cáncer en las mujeres y un 1,6\% del total de defunciones femeninas en todo el mundo. ${ }^{1}$ Cada año son recién diagnosticadas más de 1,1 millones de mujeres; que representan un $10 \%$ de todos los casos nuevos de cáncer y un $23 \%$ de todos los cánceres en las mujeres, se prevé que para el año 2010 el número

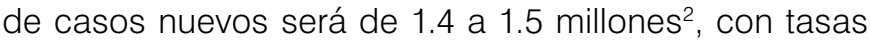
de mortalidad más altas en los países de bajos recursos cuya incidencia ha aumentando hasta un 5\%., En Amé- rica Latina y el Caribe, aproximadamente 35,000 mujeres fallecen de cáncer de mama al año. ${ }^{4}$

México no es la excepción debido a la transición demográfica se ha iniciado un proceso de envejecimiento poblacional, que aunado a la transición epidemiológica y a cambios en el estilo de vida han originado un incremento significativo de enfermedades crónico-degenerativas y neoplasias malignas como el CaMa.

En el país el CaMa afecta a mujeres adultas de cualquier nivel socioeconómico, con una variabilidad geográfica, los estados fronterizos norteños presentan las mayores tasas porcentuales del país: $24.5 \%$ del total nacional; el 45.5 por ciento ocurre en mujeres menores de 50 años a diferencia de las estadounidenses y europeas cuya edad de inicio es de 63 años. Tan solo en el periodo del 2000-2006 se registraron un total de 33,044 casos, de los cuales el $50 \%$ ocurrió en mujeres menores de 50 años ${ }^{5}$. Se estima que para el 2020 habrá 16,531 casos nuevos. ${ }^{6}$

A partir del 2006 el CaMa se ha convertido en la primera causa de mortalidad por tumores malignos, murieron 4,451 mujeres es decir un fallecimiento cada dos horas. El $60 \%$ de las mujeres que muere se ubica entre los 30 y 59 años de edad, desplazando al cáncer cervico uterino que en 1980 causaba dos veces más muertes que el cáncer de mama que hoy es la segunda causa de muerte en las mujeres de 30 a 54 años de edad. ${ }^{5}$

La mayoría de los casos es autodetectable y solo entre el 5 y el $10 \%$ se diagnostica en los estadios 0 y $\mathrm{I}^{7}$. Para el año 2000 del 40 a 50\% de los casos diagnosticados corresponde a etapas muy avanzadas (estadio III) o diseminadas (estadio IV) incrementando los costos en la atención y disminuyendo las posibilidades de curación. ${ }^{8}$ Un ejemplo de lo anterior se observó en el 2007 en el Instituto Nacional de Cancerología donde se atendieron a 744 pacientes con acceso al seguro popular, sólo el 1\% se presentó con carcinoma in situ. ${ }^{9}$

Por lo menos dos terceras partes de las muertes por cáncer de mama en mujeres menores de 75 años se pudieron evitar, esto equivale a más de 11,000 vidas si se hiciera una detección temprana y un tratamiento oportuno ${ }^{10}$.

Las mujeres que son diagnosticadas en estadio I tienen un $89 \%$ de sobrevida, y mayores probabilidades de mantenerse libres de la enfermedad a diferencia de aquéllas que son diagnosticadas en estadios más avanzados ${ }^{11}$

Las consecuencias sociales de esta problemática se pueden apreciar si se toma en cuenta que más de una quinta parte de los hogares mexicanos está dirigido por 
mujeres, son el soporte económico, responsables del cuidado y crianza de sus hijos, y muchas veces, de otros familiares, ${ }^{12}$ por lo que el diagnóstico no solo ocasiona devastadores efectos en la mujer, también en el núcleo familiar, que dada la tendencia creciente de la enfermedad permiten prever la magnitud de los daños y los desafíos que enfrentará el Sistema de Salud, ya que según estimaciones del Consejo Nacional de Población el grupo de mujeres de 25 años y más para el año 2010 y 2030 se espera que constituyan el $55.6 \%$ y $68.7 \%$, respectivamente, de la población femenina total ${ }^{13}$, constituyéndose en un grupo con alto riesgo de desarrollar la enfermedad con el que se pueden trabajar actividades de promoción de la salud de la mama, para modificación de los riesgos.

\section{FACTORES DE RIESGO}

Los factores de riesgo son las características o atributos de la mujer que incrementan la probabilidad de desarroIlar el CaMa, los dos principales son el género y la edad, otros están relacionados con la historia reproductiva, estilo de vida y algunos más de origen diverso.

\section{Historia reproductiva}

Los factores de riesgo identificados son el resultado de períodos de exposición prolongada a hormonas esteroides sexuales naturales o sintéticas, incluyen: menarquia temprana (antes de los 12 años), menopausia tardía (después de los 55 años), nuliparidad, ausencia de lactancia materna, primer embarazo a término después de los 30 , ingestión de estrógenos y/o progestinas con fines anticonceptivos o de reemplazo hormonal..$^{11,14}$

Estos factores han sido estudiados en diversos contextos y poblaciones y existe fuerte evidencia de su contribución al desarrollo del CaMa. 11,15,16,17, 18,19,20

\section{Estilo de vida}

Los estilos de vida son patrones de conducta que han sido elegidos de las alternativas disponibles para la gente, de acuerdo a su capacidad para elegir y a sus circunstancias socioeconómicas, tales como tipo de alimentación, actividad física, consumo de alcohol, tabaco u otras drogas, responsabilidad para la salud, actividades recreativas, relaciones interpersonales, prácticas sexuales, actividades laborales y patrones de consumo. ${ }^{21}$

Los aspectos del estilo de vida relacionados con el incremento en el riesgo de cáncer de mama son: consumo de alcohol, alimentación, sobrepeso u obesidad y sedentarismo.

Consumo de alcohol. Si se toman dos o más bebidas alcohólicas por día se incrementa al doble el riesgo de cáncer de mama comparado con las no bebedoras, esto es debido a que el alcohol incrementa los niveles de estrógenos en la sangre y deprime la función inmune. ${ }^{22}$

Dieta. Se ha observado mayor riesgo en aquellas mujeres con pobre consumo de frutas y vegetales, consumo excesivo de carnes rojas y de grasas saturadas. El consumo alto de carbohidratos se ha asociado a un incremento en el riesgo en mujeres mexicanas. ${ }^{23,24}$

Sobrepeso u obesidad. La Agencia Internacional de investigación sobre cáncer estima que el $25 \%$ de los casos de cáncer son causados por sobre peso u obesidad y por un estilo de vida sedentario, que aumentan el riesgo por varios mecanismos: incremento del nivel de estrógenos y testosterona, hiperinsulinemia y resistencia a la insulina, incremento en la inflamación, y depresión en la función inmune. ${ }^{25}$

Este factor en nuestro país es grave ya que de acuerdo con la Encuesta Nacional de Salud y Nutrición 2006, el $71.9 \%$ de las mujeres mayores de 20 años tienen sobrepeso $u$ obesidad. ${ }^{26}$

Diversos reportes de meta análisis han evidenciado la relación entre el índice de masa corporal y el cáncer de mama, en los que se ha demostrado que la obesidad no incrementa el riesgo en mujeres premenopáusicas, pero sí en las posmenopáusicas. ${ }^{27}$

\section{Existen otros factores, que incrementan el riesgo tales como:}

Historia personal de cáncer de mama. Una mujer con cáncer de mama tiene tres a cuatro veces mayor riesgo de un nuevo cáncer en el otro pecho que aquellas que nunca han tenido cáncer. ${ }^{22}$

Historia familiar. EL cáncer de ovario y de mama incrementan significativamente el riesgo de presentar CaMa, por lo que es importante indagarlos en 3 generaciones; algunos indicadores genéticos son el tipo de cáncer, edad de aparición, presencia de enfermedad bilateral, edad al fallecimiento ${ }^{11}$. El riesgo incrementa con el número de parientes afectados, una mujer que tiene una familiar en 
primer grado (madre, hermana, hija) tiene 2 o más veces riesgo de presentar la enfermedad antes de los 50 años y en edad más temprana que las parientes afectadas. ${ }^{28,29}$

Presencia de mutación de genes BRCA1 y BRCA2. Cerca del 5 al 10\% de todos los cánceres de mama están relacionados con mutaciones genéticas. Se han identificado 2 genes el BRCA1 y BRCA2, que están localizados en los genes 17 y 13 respectivamente, cerca del 50 a $60 \%$ de las mujeres con mutaciones en estos genes desarrollarán cáncer de mama a los 70 años de edad.15,22

\section{Antecedentes personales de enfermedad benigna.}

Cuando una mujer tiene patología mamaria benigna (proceso proliferativo, hiperplasia atípica) tiene de 4 a 5 veces mayor riesgo de desarrollar la enfermedad que aquellas mujeres que no tienen cambios proliferativos en el pecho. ${ }^{15}$

Otras variables que se asocian al riesgo elevado son número de biopsias del pecho, resultados de hiperplasia atípica o carcinoma lobular in situ, antecedentes de terapia de radiación en tórax y densidad mamaria aumentada en una mamografía. ${ }^{11}$

Aunque el CaMa puede presentarse aun sin factores de riesgo la presencia de dos o más incrementan la posibilidad de desarrollar la enfermedad por lo que es fundamental hacer una evaluación minuciosa de las mujeres.

Existen algunos modelos para predecir el riesgo de padecer cáncer de mama, el más usado es el Modelo de Gail que fue desarrollado de datos obtenidos del Breast Cancer Detection Demostration Project, en el que se determinaron los factores predictores significativos para presentar cáncer de mama con el tiempo, y entonces se estimó el riesgo relativo para cada uno de éstos. ${ }^{30,31}$

De los factores de riesgo descritos algunos no se pueden modificar como la edad, el género y la herencia, sin embargo existen otros que sí; la American Cancer Society identifica la paridad, edad del primer nacimiento, amamantamiento, anticonceptivos orales, terapia de reemplazo hormonal, obesidad, dietas altas en grasas, nivel de ejercicio y consumo de alcohol como factores de riesgo potencialmente modificables para cáncer de mama. ${ }^{20}$

En este contexto, enfermería debe enfrentar el reto de la promoción de la salud y detección temprana teniendo un rol protagónico en la coordinación de esfuerzos de las organizaciones no gubernamentales y prestadores de sa- lud, ya que a través de programas educativos las mujeres se empoderan y modifican su estilo de vida con la consecuente disminución de enfermedades crónico degenerativas, entre éstas el CaMa.

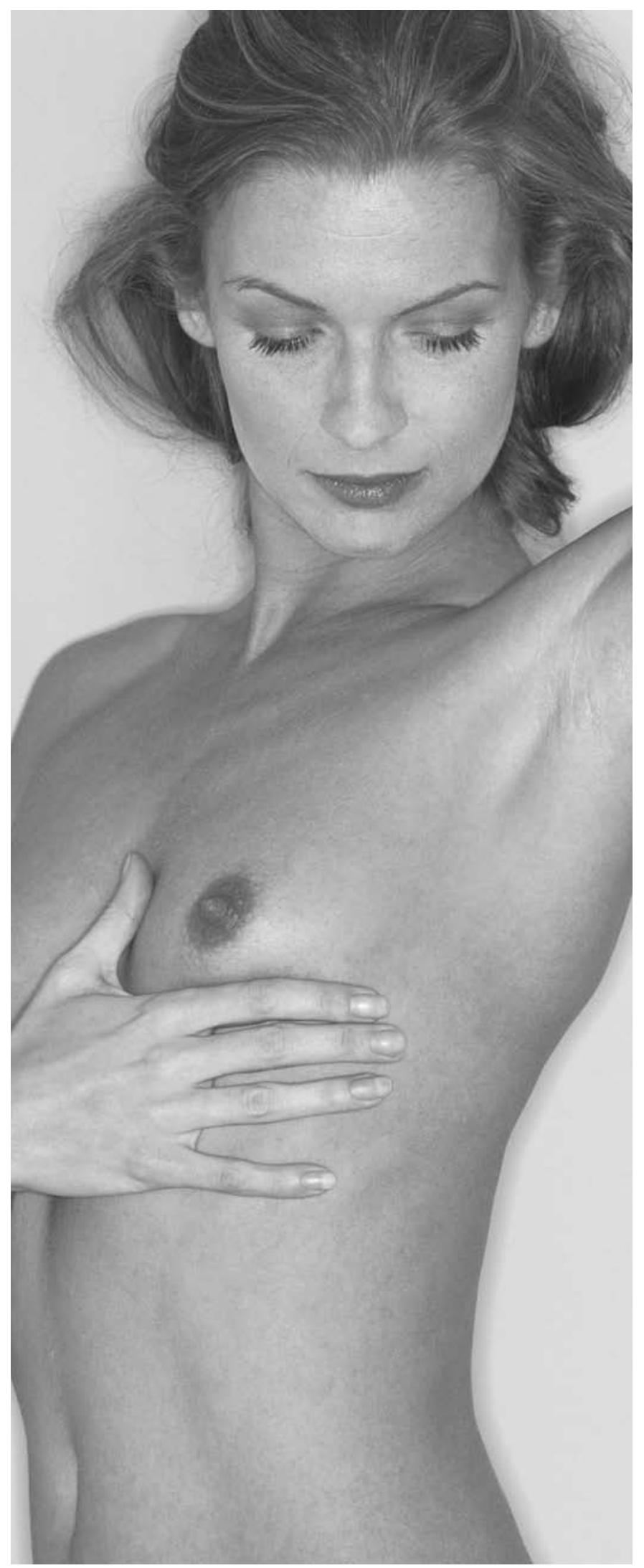




\section{Los programas educativos pueden incluir los siguientes factores:}

Ejercicio. La evidencia científica demuestra que la actividad física e intervenciones dietéticas cambian los biomarcadores para riesgo de cáncer. El ejercicio regular mejora el bienestar físico y psicológico y se asocia a otras conductas saludables como dieta, el control de peso, pero además hay suficiente certeza de que reduce la progresión del cáncer y los riesgos de los tratamientos así como las recaídas. ${ }^{32,33,34}$

Dieta. Cuando la ingesta de lípidos es adecuada y el consumo de verduras, específicamente crucíferas es abundante la dieta se convierte en un factor protector. Existe fuerte evidencia de que verduras, frutas, cereales integrales, fibra dietética, ciertos micronutrientes como los folatos, algunos ácidos grasos y la actividad física, protegen contra el CaMa. ${ }^{24}$

Aunque no hay medidas de protección específica se puede alertar a las mujeres sobre los métodos de detección: autoexploración, examen clínico por personal de salud capacitado y la mastografía.

Las enfermeras pueden dar un giro en la vida de las mujeres al incrementar los conocimientos sobre la salud de los senos, que se constituirá en una importante herramienta para empoderarlas sobre la importancia del cuidado de su salud. Además de presentarse la enfermedad, el tener conocimientos está relacionado directamente con el estadio del diagnóstico y con la sobrevida. ${ }^{35}$

Toda mujer tiene derecho a recibir educación de la salud de los senos, que debe ser culturalmente apropiada, dirigida y adaptada a cada población especifica, hay que concientizarlas sobre el papel protagónico que tienen, son ellas quienes deben estar informadas sobre el CaMa y hacer cambios en su estilo de vida para reducir peligros de padecerlo.

Es en el ámbito educativo donde las enfermeras pueden contribuir a la promoción de la salud del seno, proporcionando información veraz, oportuna, sistematizada, fortaleciendo la autoestima y el enfoque de género, para que ambos, hombres y mujeres estén bien informados de los riesgos del CaMa, que tomen mejores decisiones para el autocuidado de su salud y busquen oportunamente el apoyo y la atención de los servicios de salud en aras de tener una vida saludable.

A partir de la evaluación de los riesgos, se puede ofrecer a las mujeres un servicio de consejería para la pro- moción de la salud, prevención y modificación de riesgos. La consejería se puede constituir en espacio para la generación de conocimientos y la aplicación de modelos de atención que consideren creencias, conocimientos, conductas y habilidades de las pacientes para ofrecer un cuidado culturalmente competente.

Agradecemos la colaboración de la estudiante Verónica Gamino Villarreal en la sistematización de la literatura.

\section{REFERENCIAS BIBLIOGRÁFICAS}

1 Anderson B, Shyyan R, Eniu AE, Smith R, Yip C, Bese NS, Chow L, Masood S, Ramsey S, Carlson R.. El cáncer de mama en los países con Recursos limitados: Sinopsis de las Normativas del 2005 de la Iniciativa Mundial de Salud de la Mama. The Breast Journal 2007; 13 suppl 1:S62-82.

2 Iniciativa Mundial de Salud de Mama (Breast Health Global Initiative / BHGI) Consultado el 22 nov 08. Disponible en: http://www.paho.org/Spanish/AD/DPC/NC/pcc-bhgiabout.htm

3 Normas internacionales para la salud de mama y el control de cáncer de mama (Iniciativa Mundial de Salud de la Mama / Breast Health Global Initiative / BHGI, 2006) Consultado el 22 nov 08. Disponible en: http://www.paho.org/ Spanish/AD/DPC/NC/pcc-breast-cancer-guidelines.htm

4 Knaul FM, Lozano R, Arreola-Ornelas H, Gómez Dantés H. Cáncer de mama Tómatelo a pecho. México: Numeralia del cáncer de mama. Competitividad y Salud y Observatorio de la Salud; Fundación Mexicana para la Salud, 2008.

5 Lozano R, Knaul FM, Gómez Dantés, Arreola-Ornelas y Méndez, 2008. "Tendencias en la mortalidad por cáncer de mama en México, 1979-2006". Observatorio de la Salud. Documento de trabajo. Competitividad y Salud, Fundación Mexicana para la Salud)

6 López L, Suárez I. Cáncer de Mama. En Chávez AM, Uribe P, Núñez L, Palma Y. Coordinadores. La salud Reproductiva en México. Análisis de la Encuesta Nacional de Salud Reproductiva 2003. pp. 235-250.

7 Knaul FM, Nigenda G, Lozano R, Arreola-Ornelas H, Langer A, Frenk J. Cáncer de mama en México: Una prioridad apremiante. Salud Pública Mex 2009; 51 supl 2:S335-44.

8 Secretaría de Salud. Programa de Acción Cáncer de Mama. México, 2002 p.62

9 Mohar A, Bargallo E, Ramírez MT, Lara F, Beltrán-Ortega A. Recursos disponibles para el tratamiento de cáncer de mama en México. Salud Pública Mex 2009; 51 supl 2: S265-S269.

10 Franco-Marina F, Lozano R, Villa B, Solís P. La mortalidad en México, 2000-2004. Muertes evitables: magnitud, dis- 
tribución y tendencias. México, DF: Dirección General de Información en Salud, Secretaría de Salud, 2006.

11 Lester J. Breast Cancer in 2007: Incidence, Risk Assessment, and Risk Reduction Strategies. Clinical Journal Oncology Nursing 2007; 11(5):619-23.

12 Secretaría de Salud. CONAPO. 8 de marzo, Día Internacional de los Derechos de la Mujer Comunicado de prensa 07/05

13 Secretaría de Salud NORMA Oficial Mexicana NOM-041SSA2-2002, Para la prevención, diagnóstico, tratamiento, control y vigilancia epidemiológica del cáncer de mama. Consultada el 11 de enero del 2009. Disponible en: http:// www.salud.gob.mx/unidades/cdi/nom/041ssa202.html

14 Lacovara JE, Ray J. Deciphering the Diagnostics of Breast Cancer. Medsurg Nursing 2007; 16(6):391-410.

15 McPherson K, Steel CM, Dixon JM. Breast cancer-epidemiology, risk factors, and genetics. British Medical Journal. (International edition). London: Sep 9, 2000. Tomo 321, N 7261; pg. 624, 5 pgs.

16 Sweeney C, Blair CK, Anderson KE, Lazovich A, Folsom A. Risk Factors for Breast Cancer in Elderly Women. American J Epidemiol 2004; 160 (9): 868-875.

17 Jiménez R. Frecuencia y factores de riesgo asociados en pacientes con diagnóstico de cáncer de mama, en el Centro Nacional "La Raza" del IMSS, UMAE Hospital de Ginecología y Obstetricia No. 3 Servicio de Oncología. [Tesis] Universidad Nacional Autónoma de México; 2008; F-2008-3504-9 R-2008-3504-19:1-53.

18 Mendoza CM, Galván EA. Factores de riesgo reproductivo para cáncer de mama en pacientes atendidas en un hospital urbano de segundo nivel. Ginecol Obstet Mex. 2007; 75: 11-16.

19 Fragoso J. Mainero F. Cáncer de mama en usuarias de terapia hormonal de reemplazo estratificadas con la clasificación de Mainero. Ginecol Obstet Méx 2007;75(8):439-47.

20 Burak L; Boone B. College Women and Breast Cancer: Knowledge, Behavior, and Beliefs Regarding Risk Reduction. American Journal of Health Education 2008; 39 (4): 206.

21 López JM, Ariza CR, Rodríguez JR, Munguía C. Construcción y validación inicial de un instrumento para medir el estilo de vida en pacientes con diabetes mellitus tipo 2 . Salud Pública Méx 2003; 45:259-268.

22 McTiernan A, Gralow J, Talbott L. Breast Fitness. An optimal Exercise and Health Plan for Reducing Your Risk of Breast Cancer. New York, St. Martin's Press, 2000:16-17.

23 Romieu I, Lazcano E, Sanchez LM, Willett W, Hernandez M. Carbohydrates and the Risk of Breast Cancer among
Mexican Women. Cancer Epidemiology, Biomarkers \& Prevention 2004; 13(8):1283-9.

24 Romieu I, Lajous M. The role of obesity, physical activity and dietary factors on the risk for breast cancer: Mexican experience. Salud Publica Mex 2009; 51 suppl 2:S172S180.

25 Stephenson G, Rose D. Breast Cancer and Obesity: An Update. Nutrition and Cancer 2003; 45(1):2-17.

26 Secretaría de Salud. Programa Nacional de Salud 20072012. Por un México sano: construyendo alianzas para una mejor salud. México, 2007, p.188

27 Renehan A, Tyson M, Egger M, Heller R, Zwahlen M. Bodymass index and incidence of cancer: a systematic review and meta-analysis of prospective observational studies. The Lancet 2008 Feb 22; 371 (9612):569, 10 pgs

28 Nelson R. AACR FCPR 2008: Family History Increases Breast Cancer Risk. Medscape Medical News 2008 Consultado 8 diciembre 2008 Disponible en: http://www.medscape.com/viewarticle/584292

29 Cardenas K, Frisch K, MD. Comprehensive breast cancer screening Programs now include individual risk assessment. Postgraduate Medicine. Minneapolis: 2003; Tomo 113 (2): 34.

30 Rhodes DJ. Identifying and counseling women at increased risk for breast cancer. Mayo Clinic Proc.; 2002; $77 ; 4 ; 355-361$

31 Richardson L, Hall IJ. Diagnostic Accuracy of the Gail Model in the Black Women's Health Study. Editorial The Breast Journal 2007; 13(4):329-31.

32 Campbell K, Mc Tierman. Exercise and Biomarkers for Cancer Prevention Studies 1,2. The Journal of Nutrition Bethesda: Jan 2007 Tomo 137, No. 1S; pg. 161S, 9 pgs.

33 Friedenreich C, Orenstein M. Physical activity and cancer prevention: Etiologic evidence and biological mechanisms. The Journal of Nutrition Bethesda: Nov 2002. Tomo 132, No. 11S; pg. S3456, 9 pgs

34 Ortiz-SP, Torres G, Mainero F, Ángeles A, López AE, Lazcano $E$, Romieu I. Actividad física y riesgo de cáncer de mama en mujeres mexicanas. Consultado 10 enero 09. Disponible en http://www.infodoctor.org:8080/ uid $=18372993$

35 Harmer V. The Nurses Role in Breast Cancer Awareness $2004 ; 28: 10$

\section{CORREO PARA CORRESPONDENCIA}

Mtra. Sofía Elena Pérez Zumano:

sepzumano2@yahoo.com.mx 